\title{
Experimental evidence of librational vibrations determining the stability of calcium borohydride
}

\author{
A. Borgschulte,,${ }^{1, *}$ R. Gremaud,${ }^{1}$ A. Züttel, ${ }^{1}$ P. Martelli, ${ }^{1}$ A. Remhof, ${ }^{1}$ A. J. Ramirez-Cuesta, ${ }^{2}$ K. Refson, ${ }^{3}$ \\ E. G. Bardaji, ${ }^{4}$ W. Lohstroh, ${ }^{4}$ M. Fichtner, ${ }^{4}$ H. Hagemann, ${ }^{5}$ and M. Ernst ${ }^{6}$ \\ ${ }^{1}$ EMPA, Swiss Federal Laboratories for Materials Testing and Research, Hydrogen \& Energy, CH-8600 Dübendorf, Switzerland \\ ${ }^{2}$ ISIS Facility, Rutherford Appleton Laboratory, Chilton, Didcot, Oxon OX11 OQX, United Kingdom \\ ${ }^{3}$ CSED, Rutherford Appleton Laboratory, Chilton, Didcot, Oxon OX11 0QX, United Kingdom \\ ${ }^{4}$ Karlsruhe Institute of Technology, Institute of Nanotechnology, D-76021 Karlsruhe, Germany \\ ${ }^{5}$ Département de Chimie Physique, Université de Genève, CH-1211 Geneva 4, Switzerland \\ ${ }^{6}$ ETH Zürich, Physical Chemistry, CH-8093 Zürich, Switzerland \\ (Received 6 October 2010; published 25 January 2011)
}

\begin{abstract}
The high energy of hydrogen vibrations in solids is the origin of their strong impact on thermodynamic properties. The peculiar structure of complex hydrides amplifies this impact. We shed light on the vibrational properties of three allotropes of $\mathrm{Ca}\left(\mathrm{BH}_{4}\right)_{2}$ using density-functional theory calculations, infrared spectroscopy, and inelastic neutron scattering. We show that the vibrational properties of $\mathrm{Ca}\left(\mathrm{BH}_{4}\right)_{2}$ depend on the specific phase and are hitherto the origin of their differences in stability.
\end{abstract}

PACS number(s): 63.20.-e, 61.05.fg, 64.60.Ej, 64.70.kt

\section{INTRODUCTION}

In the search of hydrogen-storage materials, numerous hydrogen-containing compounds have been investigated with a particular emphasis on solving the crystal structure of the compounds. The crystal structure sets the physical properties of the compound under investigation, which eventually define its use as a hydrogen storage material. The knowledge of crystal structures is of particular importance for theoretical modeling by, e.g., density-functional theory (DFT). Recently borohydrides have received a lot of attention because of their high gravimetric storage density up to 18 mass $\%$ hydrogen. ${ }^{1}$ Storage capacity, thermodynamic stability, and sorption rate are the most important properties of hydrogen storage materials and are intensively studied, but in particular the slow sorption kinetics of complex hydrides is an unsolved problem. ${ }^{1,2}$ In this respect, $\mathrm{Ca}\left(\mathrm{BH}_{4}\right)_{2}$ has potential because reversible hydrogen desorption and absorption at relatively moderate conditions have been demonstrated. ${ }^{3,4}$ Similar to many other borohydrides, $\mathrm{Ca}\left(\mathrm{BH}_{4}\right)_{2}$ shows a variety of structures depending on the synthesis and temperature of the samples. ${ }^{5-7}$ This complicates the characterization of the samples and the understanding of thermodynamics and kinetics of hydrogen sorption in this material. Furthermore, prediction of the thermal properties by an ab initio method is extrapolation from calculations at $0 \mathrm{~K}$, with high uncertainty due to the complications from entropy effects at higher temperature (see, e.g., Ref. 8). In the case of $\mathrm{Ca}\left(\mathrm{BH}_{4}\right)_{2}$, calculations of the formation energy for the various phases indicate that the formation energy of the $\alpha$ phase is only marginally lower than that of the $\beta$ and $\gamma$ phases, confirming that the $\alpha$ and $\beta$ phases of $\mathrm{Ca}\left(\mathrm{BH}_{4}\right)_{2}$ can both be present in the compound at the same thermodynamic conditions as experimentally observed. Interestingly, the density and disorder are different in the phases, evidence that the phase transition is driven by entropy. DFT calculations show that for low-lying frequencies the phonon states are different in both phases. ${ }^{9}$ At higher temperatures, different phonon states are occupied, which contribute to the entropy of the system. This effect had been anticipated on the basis of DFT calculations ${ }^{9}$ but not yet experimentally validated. The phase transformations are partly irreversible, and the phases remain stable after quenching to the measurement temperature. This enables us to measure the inelastic neutron-scattering (INS) spectra for the various phases at the same temperature $(15 \mathrm{~K})$ avoiding the change of spectra due to thermal effects (Debye-Waller-factor etc.). With this we are able to experimentally confirm the theoretical prediction of vibrations being the origin of different entropy of the allotropes.

\section{EXPERIMENT}

$\gamma-\mathrm{Ca}\left(\mathrm{BH}_{4}\right)_{2}$ was synthesized similarly to a method described in Ref. 10: $\mathrm{CaH}_{2}$ (which was ball-milled for $1 \mathrm{~h}$, at $600 \mathrm{rpm}$, in a tempered steel system) was introduced to triethylamine borazane complex, $\mathrm{Et}_{3} \mathrm{~N} \cdot \mathrm{BH}_{3}$ (Katchem Ltd.). The mixture was heated under reflux for $2 \mathrm{~h}$ at $80^{\circ} \mathrm{C}, 1 \mathrm{~h}$ at $95^{\circ} \mathrm{C}, 1 \mathrm{~h}$ at $120^{\circ} \mathrm{C}$, and finally $4 \mathrm{~h}$ at $140^{\circ} \mathrm{C}$. After cooling down to room temperature, dried and degassed n-hexane was introduced to the white solid. The latter was reduced to small pieces by means of a spatula. The suspension was stirred overnight, filtered under Ar, washed with n-hexane, and dried at room temperature under vacuum, followed by drying at $160^{\circ} \mathrm{C}$ for $16 \mathrm{~h}$. The isolated yield (with respect to $\mathrm{CaH}_{2}$ ) was $85 \%$. This procedure yields the $\gamma$ phase (see Fig. 3). Quenching after annealing at $180^{\circ} \mathrm{C}$ for $6 \mathrm{~h}$ and at $300^{\circ} \mathrm{C}$ for 5 min yields the $\alpha$ and $\beta$ phases, respectively (Fig. 3).

Nuclear magnetic resonance measurement (NMR) have been performed with the DMX-400 spectrometer (Bruker Biospin, Fällanden, Switzerland) with a field of $9.39 \mathrm{~T}$ resulting in a resonance frequency of ${ }^{11} \mathrm{~B}$ at $128 \mathrm{MHz}$. To increase the resolution, the sample was spun at $10,000 \mathrm{~s}^{-1}$ at the "magic angle" of $54.74^{\circ}$. Further improvement was achieved by decoupling the ${ }^{11} \mathrm{~B}$ signal from the ${ }^{1} \mathrm{H}$ signal.

INS spectra have been recorded at the TOSCA spectrometer of the ISIS facility of the Rutherford Appleton Laboratory in Didcot, UK. The measurements were carried out in neutron energy-loss mode on ${ }^{11} \mathrm{~B}$-enriched samples to avoid the high neutron absorption of ${ }^{10} \mathrm{~B}$ present in natural boron. The powder was mounted into a flat aluminum sample holder of $1 \mathrm{~mm}$ 
thickness. The raw data were corrected to obtain the scattering function $S(Q, \omega)$ using standard routines available at ISIS. ${ }^{11}$

The vibrational properties of the three allotropes of $\mathrm{Ca}\left(\mathrm{BH}_{4}\right)_{2}$ were calculated with $a b$ initio lattice dynamics based on density-functional theory and the plane-wave pseudopotential method as implemented in the CASTEP code. ${ }^{12,13}$ Pseudopotentials were of the optimized norm-conserving variety $^{14}$ with a plane-wave cutoff of $450 \mathrm{eV}$. Calculations were performed under the PBE approximation to exchange and correlation. ${ }^{15}$ Brillouin-Zone integration was performed according to the Monkhorst-Pack scheme with a $2 \times 3 \times 2$ mesh of $k$-points, which gave convergence of all modes to a precision of better than $3 \mathrm{~cm}^{-1}$. Pseudopotential errors in the frequencies were estimated at no more than $1 \%$ from a comparison of alternative pseudopotentials. The normal modes of the crystalline phase were determined from dynamical matrices calculated using density-functional perturbation theory. ${ }^{13,16}$ The calculated INS spectra were generated using the a Climax program. ${ }^{17}$

\section{RESULTS AND DISCUSSION}

\section{A. Calculations}

In the following we describe the calculated physical properties of $\mathrm{Ca}\left(\mathrm{BH}_{4}\right)_{2}$. As shall we see, the findings, in particular the stability of the various phases, are in perfect agreement with previous theoretical results by Majzoub and Rönnebro ${ }^{18}$ and Frankcombe et al. ${ }^{19}$ However, the interpretation of the experimental vibrational properties requires a complete data set of the fundamental properties of the systems (density of states, etc.). Furthermore, the calculations define the line of argumentation to experimentally evidence the influence of librational vibrations on the stability of calcium borohydride and are thus shortly given here.

The calculated crystal structures of $\alpha-\mathrm{Ca}\left(\mathrm{BH}_{4}\right)_{2}, \quad \beta$ $\mathrm{Ca}\left(\mathrm{BH}_{4}\right)_{2}$, and $\gamma-\mathrm{Ca}\left(\mathrm{BH}_{4}\right)_{2}$ are in very good agreement with the experimentally determined ones ${ }^{5,9}$ with lattice parameters listed in Table I. The space groups of the three phases ( $F d d d, P 4_{2} / m$, and $P b c a$ ) have a common supergroup, namely, $I 4 / \mathrm{mmm}$, which can be reached in two or three steps of structural rearrangements, so there is no simple order parameter to describe these transitions. The transitions are therefore first-order transitions. The static DFT total energy difference at $T=0 \mathrm{~K}$ between the $\alpha$ - and $\beta$-Ca( $\left(\mathrm{BH}_{4}\right)_{2}$ phases is $89 \mathrm{meV} /\left(\mathrm{Ca}\left(\mathrm{BH}_{4}\right)_{2}\right)$, and the total energy difference between the $\alpha$ and $\gamma$ phases is $35 \mathrm{meV} /\left[\mathrm{Ca}\left(\mathrm{BH}_{4}\right)_{2}\right]$.

TABLE I. Calculated (at $0 \mathrm{~K}$, this work) and experimental (at room temperature, from Ref. 5) lattice parameters of the $\alpha$ phase (space group $F d d d$ ), $\beta$ phase (space group $P 4_{2} / m$ ), and $\gamma$ phase (space group $\mathrm{Pbca}$ ) of $\mathrm{Ca}\left(\mathrm{BH}_{4}\right)_{2}$. For more information see cif-files attached as supplemental information. ${ }^{20}$

\begin{tabular}{lccll}
\hline \hline Phase & $\mathrm{a}(\AA)$ & $\mathrm{b}(\AA)$ & $\mathrm{c}(\AA)$ & Cell angles \\
\hline$\alpha$ (cal) & 8.791100 & 13.137100 & 7.5000 & $\alpha=\beta=\gamma=90^{\circ}$ \\
$\alpha$ (exp) & $8.7461(8)$ & $13.0942(9)$ & $7.4660(7)$ & $\alpha=\beta=\gamma=90^{\circ}$ \\
$\beta$ (cal) & 6.947000 & 6.947000 & 4.366000 & $\alpha=\beta=\gamma=90^{\circ}$ \\
$\beta$ (exp) & $6.9468(1)$ & $6.9468(1)$ & $4.3661(1)$ & $\alpha=\beta=\gamma=90^{\circ}$ \\
$\gamma$ (cal) & 13.0580 & 8.3880 & 7.5110 & $\alpha=\beta=\gamma=90^{\circ}$ \\
$\gamma$ (exp) & $13.0584(8)$ & $8.3881(4)$ & $7.5107(4)$ & $\alpha=\beta=\gamma=90^{\circ}$ \\
\hline \hline
\end{tabular}

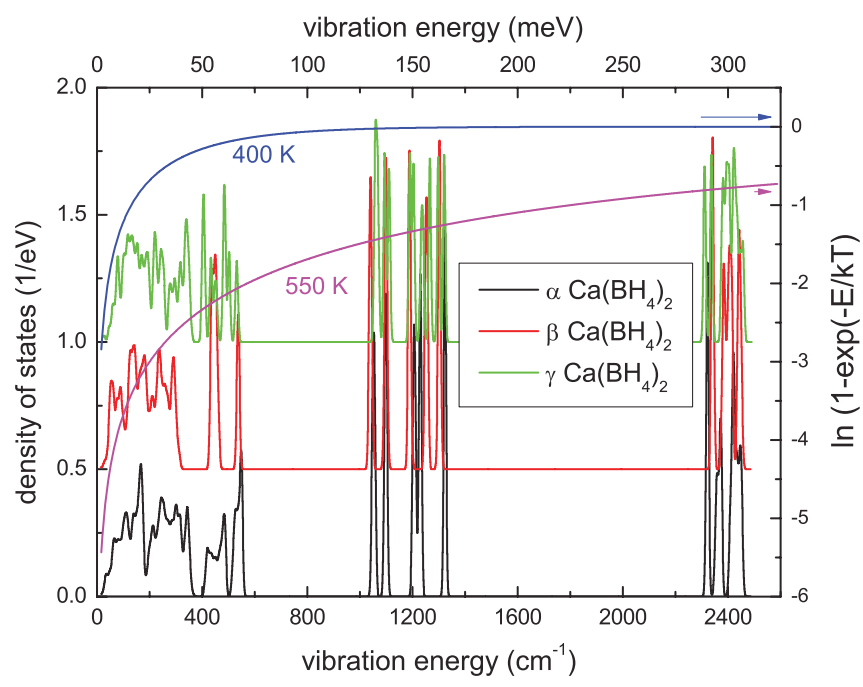

FIG. 1. (Color online) Calculated density of phonons states $g(E)$ of the three polymorphs of $\mathrm{Ca}\left(\mathrm{BH}_{4}\right)_{2}$. The continuous lines are plots of the function $\ln \left(1-e^{\frac{-E}{k T}}\right)$ with $T=400,550 \mathrm{~K}$ illustrating the impact of the partition function on the free energy of the system.

The values are in fair agreement with previous publications [Lee et al. ${ }^{9} \Delta H(\alpha-\beta)=113 \mathrm{meV}$, Buchter et al.: ${ }^{5}$ $\Delta H(\alpha-\beta)=135 \mathrm{meV}, \Delta H(\alpha-\gamma)=63 \mathrm{meV}]$. Considering only the enthalpy differences, the $\alpha$ phase is the most stable polymorph, followed by the $\gamma$ and $\beta$ phases. The observed reversible transition from the $\alpha$ to the $\beta$ phase is thus based on the additional entropy terms, eventually giving the Helmoltz free energy $F .^{21,22}$ Because the borohydrides are assumed to be stoichiometric compounds, ${ }^{23}$ the configurational entropy is negligible, and the dominant term is the quasiharmonic phonon contribution. The occupation of the phonons depends on temperature, which in turn gives the free energy of the phonons $F_{\mathrm{vib}}:^{22}$

$$
\begin{aligned}
F_{\mathrm{vib}} & =\sum_{j=1}^{3 N} \sum_{q} k T \ln \left(1-e^{\frac{-\hbar \omega_{j}}{k T}}\right) \\
& =k T \int d E \cdot g(E) \ln \left(1-e^{\frac{-E}{k T}}\right),
\end{aligned}
$$

where $E=\hbar \omega$ is the energy of the vibrations. The second equation is used to depict the effect of the density of phonon states $g(E)$ on the free energy. The calculated density of phonon states $g(E)$ is plotted for the three polymorphs of $\mathrm{Ca}\left(\mathrm{BH}_{4}\right)_{2}$ in Fig. 1. Significant differences of the density of phonon states are observed at low energies. However, at high temperatures these states contribute the most to $F_{\text {vib }}$ due to the term $\ln \left(1-e^{\frac{-E}{k T}}\right)$. The impact of low-energy phonons (about $50 \mathrm{meV}$ ) on the free energy is particularly different between 400 and $550 \mathrm{~K}$ (the temperature range of the phase transitions), depicting the origin of the vibrational entropy-driven phase transition.

Figure 2 shows the resulting free energy of the three polymorphs from DFT calculations including static total energy, zero-point energies, and quasiharmonic phonon contributions. The $\alpha$ phase is most stable up to approximately $550 \mathrm{~K}$, when the $\beta$ phase becomes most stable in good agreement with the observed phase transition ${ }^{5}$ and with previous theoretical 


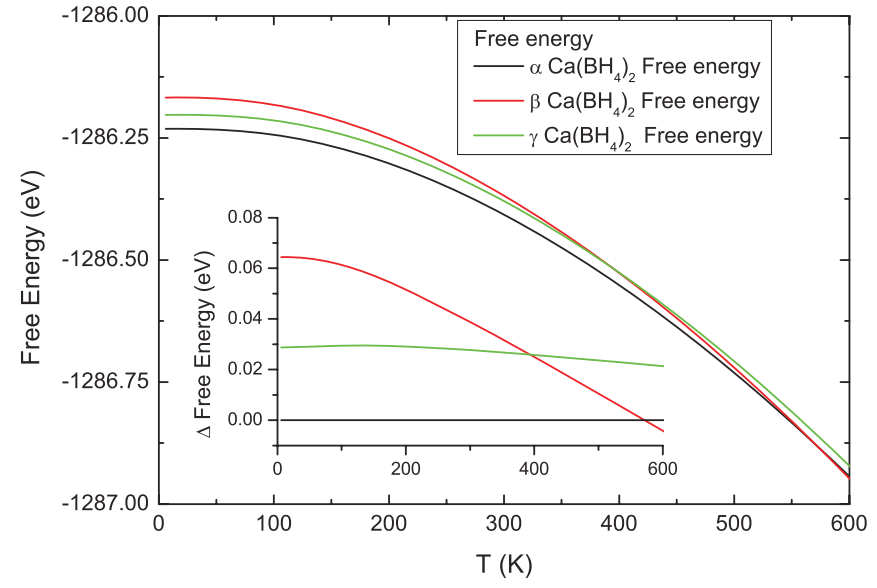

FIG. 2. (Color online) Calculated free energy $F$ of the three polymorphs of $\mathrm{Ca}\left(\mathrm{BH}_{4}\right)_{2}$ as a function of temperature. The inset shows the difference in free energy with respect to the $\alpha$ phase.

findings. ${ }^{18}$ The $\gamma$ phase is not energetically favored at any temperature, giving the theoretical explanation of the metastability of this phase. The $\gamma$ phase is formed during crystallization from solution. Due to kinetic constraints, it remains in this crystal structure up to a temperature at which the mobility of the ions is high enough to allow for rearrangement into the energetically favored $\alpha$ phase. A back-transformation is then not possible, because at a higher temperature the $\beta$ phase is energetically favored. There have been reports of various other phases occurring during heating and desorption cycles. ${ }^{6,24}$ It is likely that these phases are metastable intermediates as is $\gamma-\mathrm{Ca}\left(\mathrm{BH}_{4}\right)_{2}$. Their existence depends on details of the experimental procedure (preparation ${ }^{5}$ and resulting impurity concentrations, ${ }^{25}$ temperature, annealing time, hydrogen back pressure, etc.) and are thus not considered here.

\section{B. Experiments}

The structure transformations are experimentally followed by x-ray diffraction (see Fig. 3). The diffraction pattern of

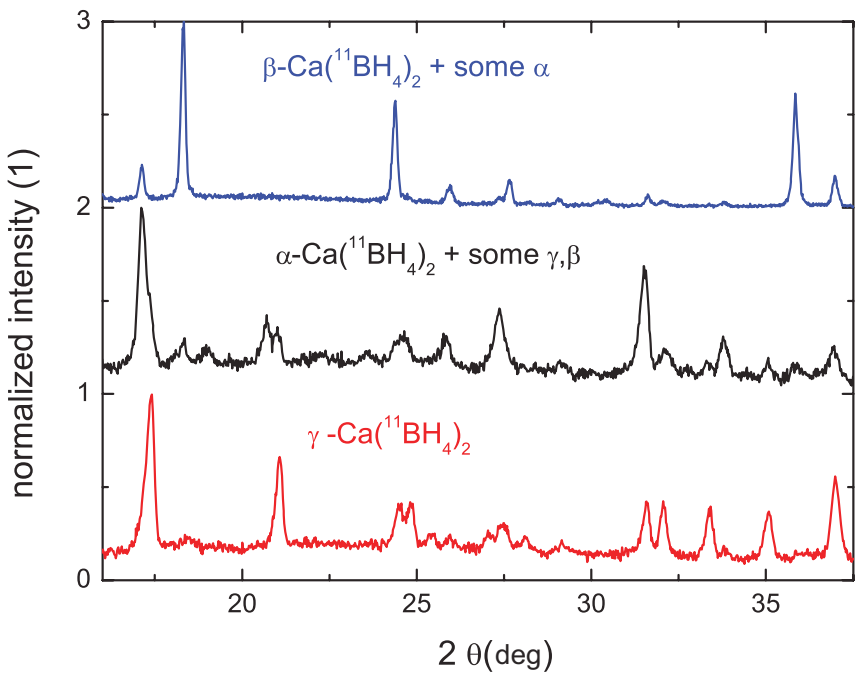

FIG. 3. (Color online) X-ray diffraction of $\mathrm{Ca}\left(\mathrm{BH}_{4}\right)_{2}$ as prepared ( $\gamma$ phase) and after heat treatments yielding the $\alpha$ and $\beta$ phases.

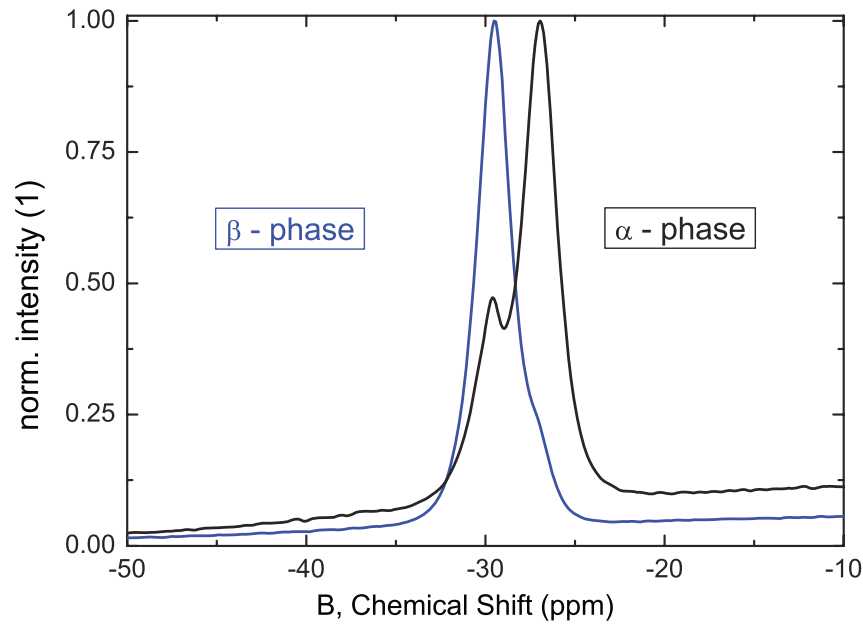

FIG. 4. (Color online) Normalized NMR spectra $\left({ }^{11} \mathrm{~B}\right.$ resonance line) of the $\alpha$ and $\beta$ phases of $\mathrm{Ca}\left(\mathrm{BH}_{4}\right)_{2}$.

the as-prepared samples shows the pure $\gamma$ phase. After annealing steps, the sample contains mainly $\alpha$ - and $\beta$-Ca(BH $\left(\mathrm{BH}_{4}\right)_{2}$, respectively, with impurities of the corresponding other polymorphs. The impurities are due to the small energy difference; the sample modifications are called in this paper the $\gamma, \alpha$, and $\beta$ phase, respectively.

As a consequence of the structure changes, the environment of the atoms is altered. An experimental technique to probe the chemical environment of an atom is NMR. NMR spectra $\left({ }^{11} \mathrm{~B}\right.$ resonance line) show a distinct chemical shift of the $\alpha$ and $\beta$ phases of $\mathrm{Ca}\left(\mathrm{BH}_{4}\right)_{2}$ (Fig. 4). In addition, the different phases have different spin-lattice relaxation times, which can be measured using inversion-recovery experiments (to be published elsewhere).

It is aim of this paper to investigate the vibrational structure responsible for the peculiar phase transformations. Several papers report on infrared (IR) and Raman spectra of $\mathrm{Ca}\left(\mathrm{BH}_{4}\right)_{2}$. However, for experimental reasons, these data focus mainly on the internal modes of the borohydrides, while, as we have shown, the modes below $1000 \mathrm{~cm}^{-1}$ contribute to the free energy in the temperature range of interest. Inelastic neutron scattering, on the other hand, is particularly sensitive in the low-energy range. INS is based on the scattering of neutrons with phonons. Because of averaging over a large momentum, the scattering power $S(Q, \omega)$ is an effigy of the phonon density of states ("amplitude and cross section weighted density of states"): ${ }^{11}$

$$
S(Q, \omega)=Q^{2}[n(\omega, T)+1] \cdot G(\omega) / \omega,
$$

where $G(\omega)$ is the multiphonon density of states and $n(\omega, T)$ is the Bose factor. The latter factor is temperature dependent, and thus all samples are measured at the same temperature of $15 \mathrm{~K}$. The scattering cross section of hydrogen is particularly large, and thus $S(Q, \omega)$ is the partial phonon density of states of hydrogen in first order. The spectra include multiphonon processes, which makes a conversion of $G(\omega)$ into the true density of states $g(\omega)$ difficult. Instead, we compare the experimental spectra $S(Q, \omega)$ with the calculated scattering power based on DFT calculations and the aClimax program, ${ }^{17}$ which include the multiphonon processes and the instrumental 


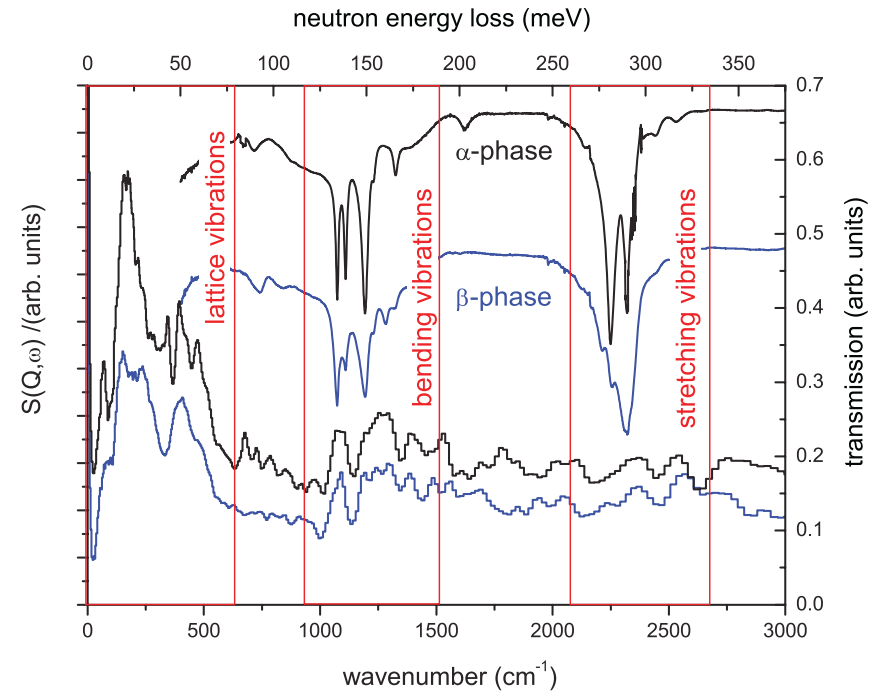

FIG. 5. (Color online) INS at $T=15 \mathrm{~K}$ (bottom curves) and IR spectra at $T=300 \mathrm{~K}$ (top curves) of the $\alpha$ and $\beta$ phases, respectively.

resolution. Peaks in the spectra correspond to the high density of states, often due to small dispersion of lattice vibrations at the Brillouin zone boundaries and/or the zone center. In addition, we have the low-energy partial hydrogen phonon density of states $G(\omega)$ from Eq. (2) after substraction of the multiphonon scattering $S(Q, \omega)$ using the iterative procedure published in Ref. 26.

Figure 5 shows the INS and IR spectra of $\mathrm{Ca}\left({ }^{11} \mathrm{BH}_{4}\right)_{2}$ in the $\alpha$ and $\beta$ phases. The spectra can be schematically divided into three regions. At the lowest energies are the translational lattice vibrations, where the whole $\mathrm{BH}_{4}^{-}$moves against the $\mathrm{Ca}^{+}$ ion. Phonons originating from librational motions-also called constrained rotations - of the $\mathrm{BH}_{4}^{-}$units appear at slightly higher energies, e.g., as a large band around $50 \mathrm{meV}$ in the INS spectrum. Internal vibrations of the $\mathrm{BH}_{4}^{-}$unit are found around $150 \mathrm{meV}$ (B-H bending) and $300 \mathrm{meV}$ (B-H stretching). As we have discussed, the sensitivity and resolution of INS are inferior to optical spectroscopy at higher energies. The investigation of internal modes is thus easier with the help of Raman and IR spectroscopy. The change of these spectra are due to the change of the crystal symmetry activating or deactivating an IR or Raman transition. ${ }^{24,27}$ The total density of states of these phonons is less affected by the crystal structure, as, e.g., is visible for the bending modes. A difficulty of INS is the occurrence of strong overtones (multiphonon scattering), particularly at higher energies. These overtones also occur in the IR and Raman spectra; however, their identification is easier than in the INS spectra. In this paper we want to focus on the phonon states responsible for thermodynamic properties. With energies of above $150 \mathrm{meV} \simeq 1730 \mathrm{~K}$, bending and stretching modes have a minor influence on thermodynamic changes at temperatures of less than the experimentally observed $600 \mathrm{~K}$ (compare Fig. 1). The impact on thermodynamics can thus originate only from lattice vibrations. The low-energy part comprising translational and librational vibrations indeed show rich structures and significant differences between the three allotropes. As INS is an effigy of the hydrogen phonon density of states, and keeping in mind that hydrogen-derived vibrations have a high density of states when compared to
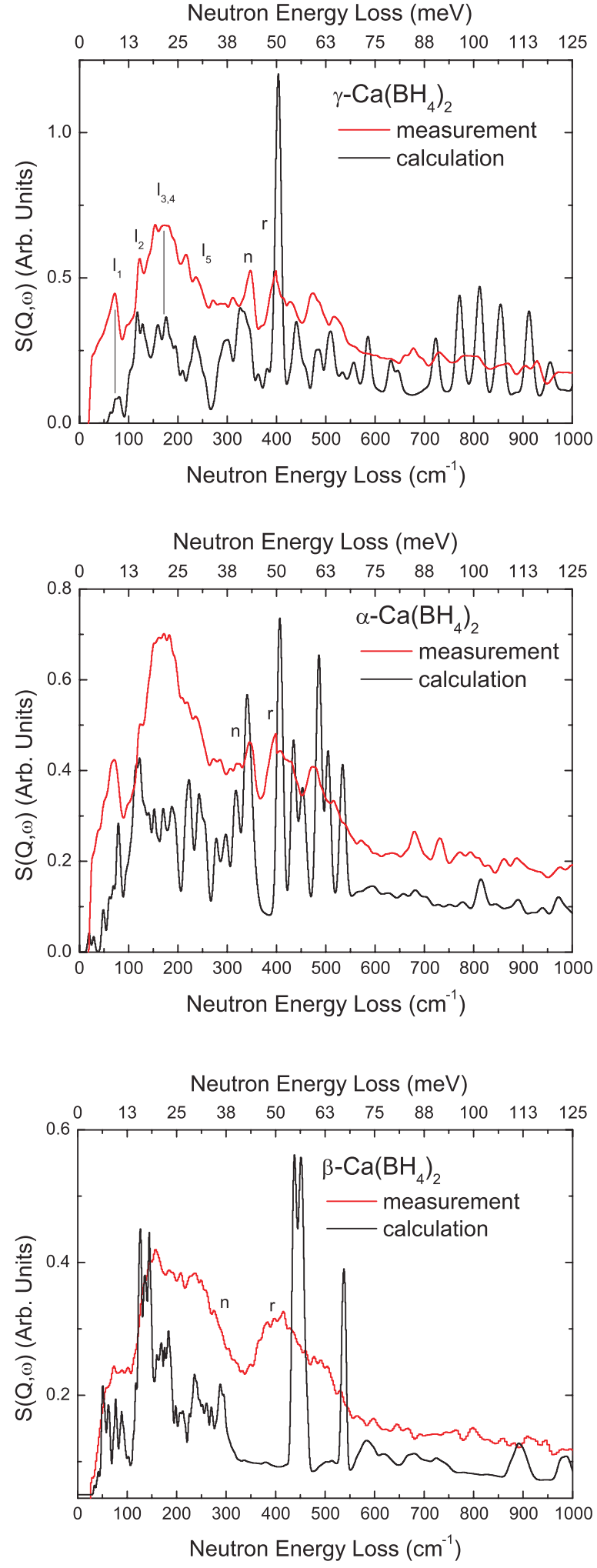

FIG. 6. (Color online) INS spectra as measured and as calculated of the $\gamma, \alpha$, and $\beta$ phases, respectively.

the other atoms involved, the differences in the INS spectra are directly related to the vibrations contributing to the free energy and hitherto to the stability of the allotropes.

Figure 6 shows INS spectra as measured and as calculated for the $\gamma, \alpha$, and $\beta$ phases, respectively. Significant intensity 
TABLE II. Comparison of most prominent librational modes $n, r$ (in $\mathrm{cm}^{-1}$ ).

\begin{tabular}{lcccc}
\hline \hline Phase & $n$ Exp & $n$ Theo & $r$ Exp & $r$ Theo \\
\hline$\alpha-\mathrm{Ca}\left(\mathrm{BH}_{4}\right)_{2}$ & 345 & 340 & 396 & 406 \\
$\beta-\mathrm{Ca}\left(\mathrm{BH}_{4}\right)_{2}$ & $272 / 286$ & $282 / 294$ & $379 / 407$ & $440 / 451$ \\
$\gamma-\mathrm{Ca}\left(\mathrm{BH}_{4}\right)_{2}$ & 346 & 336 & 397 & 402 \\
\hline \hline
\end{tabular}

from lattice vibrations is calculated and observed up to an energy of about $70 \mathrm{meV}$, which is in agreement with the first INS data for this compound. ${ }^{28}$ However, there is no quantitative agreement of the measurement with the calculated data. On the other hand, prominent structures in the experimental spectra, e.g., the peaks assigned with $l_{i},(i=1,2, \ldots)$ and $n$, $r$ in Fig. 6, are in good agreement with the calculated ones. The $\alpha$ phase and $\beta$ phase as prepared in this way are not pure phases containing some of the corresponding other phases of $\mathrm{Ca}\left(\mathrm{BH}_{4}\right)_{2}$ (see Fig. 3). The spectrum is thus a superposition of the corresponding INS spectra of all three phases. This can partly explain some deviations of the $\alpha$ phase and the $\beta$ phase. However, the $\gamma$ phase is pure, but particularly at lower energy the measured intensity is significantly higher than calculated. These deviations may be due to nonperfect samples (disorder, impurities) and multiphonon scattering. Considering the contribution of multiphonon neutron scattering as shown in Fig. 8 shifts the curves and leads to a better agreement. However, a calculation of multiphonon contribution ${ }^{29}$ as, e.g., conducted in $\mathrm{AlH}_{3}$ to derive the density of phonon states (see Ref. 30) may be error prone due to the many transitions. Further discussions will thus concentrate on changes of the spectra and on the energy of the modes $n, r$. Although INS maps the density of states, phonon states with low dispersion (e.g., internal modes, but also the librational modes) may be assigned. For the low-energy part, the hydrogen phonon density of states as derived from the corrected scattering function using an iterative procedure ${ }^{26}$ is also given in Fig. 8. From an analysis of the vibrational structure (see also Lee et al. in Ref. 9, and Refs. 24,31), the $n$ and $r$ vibrations are identified as librational modes by comparison of experiment with theory and summarized in Table II. For one $\mathrm{BH}_{4}$ group in $\mathrm{T}_{d}$ symmetry, the rotation transforms as $\mathrm{T}_{1}$. In $\mathrm{C}_{2}$ symmetry, this becomes $\mathrm{A}+\mathrm{B}+\mathrm{B}$. In the crystal, $\mathrm{A}$ becomes $\mathrm{A}_{g}+$ $\mathrm{B}_{2 g}+\mathrm{A}_{u}+\mathrm{B}_{2 u}$, and $\mathrm{B}$ becomes $\mathrm{B}_{1 g}+\mathrm{B}_{1 u}+\mathrm{B}_{2 g}+\mathrm{B}_{2 u}$. Taking the results of Miwa et al. ${ }^{31}$ who give the symmetry of the calculated lattice modes (for the $\alpha$ phase, i.e., $F d d d$ ), one obtains basically three distinct branches (see Table III).

TABLE III. Symmetry analysis of libration modes using results of Miwa et al..$^{31}$ (all frequencies in $\mathrm{cm}^{-1}$ ).

\begin{tabular}{lcc}
\hline \hline $\mathrm{A}_{g}$ & 211 & 492 \\
$\mathrm{~A}_{u}$ & 254 & 386 \\
$\mathrm{~B}_{2 g}$ & 245 & $479(+169)$ \\
$\mathrm{B}_{2 u}$ & $206-296$ & $406-412$ \\
$\mathrm{~B}_{1 g}$ & 298 & $521(+97,156,257)$ \\
$\mathrm{B}_{3 g}$ & 291 & $529(+43,101,339)$ \\
$\mathrm{B}_{1 u}$ & $488-496$ \\
$\mathrm{~B}_{3 u}$ & $292-328$ & $514-524$ \\
\hline \hline
\end{tabular}

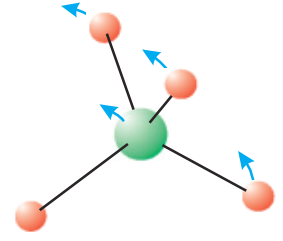

$n$-vibration

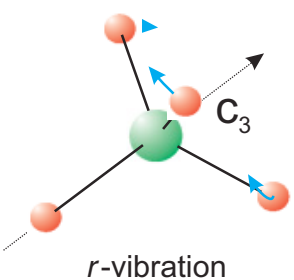

$r$-vibration
FIG. 7. (Color online) Simplified visualization of the librational modes $n$ and $r$. The $n$-vibrations may be described by a "nick" movement with one hydrogen atom standing still, and the $r$-vibrations by a constrained rotation around the $c_{3}$ axis. The complete movements of all atoms involved are visualized as movies found in the Supplemental Information. ${ }^{20}$

The large calculated LO-TO splitting of the lower mode is remarkable and may possibly lead to smearing out in the vibrational density of states. So the band with the letter $n$ is the first B mode (calculated by Miwa et al. ${ }^{31}$ around $300 \mathrm{~cm}^{-1}$ ), and the $r$ band around $400 \mathrm{~cm}^{-1}$ to the $\mathrm{A}_{u}$ and $\mathrm{B}_{2 u}$ component of the A mode. The band around $500 \mathrm{~cm}^{-1}$ is also a librational mode, but in this case its energy is too high to be significantly thermally populated.

The names were chosen according to the characteristic movements of the $\mathrm{BH}_{4}$ units as illustrated in Fig. 7 (see also movies in the Supplemental Information). ${ }^{20}$ The $n$-vibrations may be described by a "nick" movement, and the $r$-vibrations by a constrained rotation around the $c_{3}$ axis. These librational modes are present in all three phases but differ in energy.

The differences between the $\alpha$ and $\gamma$ phases are relatively small, while both attributed lines shift significantly for the $\beta$ phase. The energy range of these vibrations corresponds to a temperature of around $550 \mathrm{~K}$, at which the phase transformations occur. For illustration, the energy equivalent of 400 and $550 \mathrm{~K}$ is added in Fig. 8 comparing the INS spectra of the $\beta$ and $\gamma$ phases. Although the agreement between experiment and calculation is only semiquantitative, the

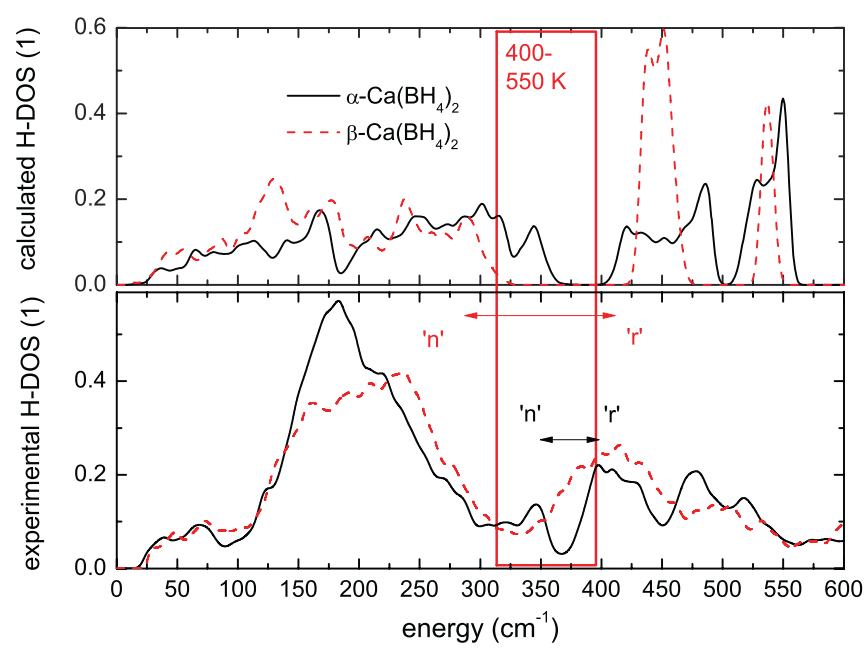

FIG. 8. (Color online) Comparison of the hydrogen phonon density of states of the stable polymorphs of $\mathrm{Ca}\left(\mathrm{BH}_{4}\right)_{2}$, i.e., $\alpha$ and $\beta$ phases, respectively, as measured (bottom curves) and as calculated (top curves). The opening of the gap between the two characteristic librational modes $n$ and $r$ is indicated. 
opening of the gap between the two characteristic librational modes is evident. Librational modes depend sensitively on the environment of the tetrahedrons in a gear-like fashion. While the next-neighbor distances of B-H, B-B, and Ca-B do not differ significantly between the phases, the orientations of the tetrahedral $\mathrm{BH}_{4}^{-}$-units change dramatically with the crystal structure (see, e.g., Fig. 3 in Ref. 5). Consequently, the crystal potential is altered as experimentally shown by NMR (compare Fig. 4 ) and changes the energy of the vibrations. In a simplified picture, at high temperatures, the $\mathrm{BH}_{4}^{-}$-units vibrate in a way to minimize the free energy of the system by aiming for a crystal structure with a minimum influence of the librational modes.

\section{CONCLUSION}

The phase transformation in $\mathrm{Ca}\left(\mathrm{BH}_{4}\right)_{2}$ has been investigated by means of vibrational spectroscopy combined with density functional theory. Previous theoretical work proposed that the peculiar vibrational structure is the origin of the various phase transformations in this system. From ab inito calculations of the various phases we derived the Helmholtz free energies, which depend significantly on the quasiharmonic phonon contribution. The corresponding phonons were measured using inelastic neutron scattering, which are in good agreement with the calculated spectra derived from DFT. The measurements give direct evidence for librational modes as the origin of the phase transformations in this system.

The effect has consequences on the anticipation of novel hydrogen storage materials. The energy differences from vibrational entropy are of the order of $16 \mathrm{meV} / \mathrm{H}_{2}$, which is not negligible when compared to the optimum heat of formation of $420 \mathrm{meV} / \mathrm{H}_{2}$ required for application. Consequently, the impact of vibrational entropy should be carefully taken into account for the calculation of complex hydrides.

\section{ACKNOWLEDGMENTS}

This work was financially supported by the European Commission (NESSHY Contract No. 518271), the HGF initiative "FuncHy," the Swiss National Science Foundation (SNF-Project 200021-119972/1), and the Swiss Federal Office of Energy. Computing resources were provided by STFC's e-Science facility, SCARF. *andreas.borgschulte@empa.ch

${ }^{1}$ A. Züttel, A. Borgschulte, and S. I. Orimo, Scr. Mater. 56, 823 (2007), and references therein.

${ }^{2}$ U. Eberle, M. Felderhof, and F. Schüth, Angew. Chem. Int. Ed. 48, 6608 (2009).

${ }^{3}$ J.-H. Kim, S.-A. Jin, J.-H. Shim, and Y. W. Cho, Scr. Mater. 58, 481 (2008).

${ }^{4}$ C. Rongeat, V. D'Anna, H. Hagemann, A. Borgschulte, A. Züttel, L. Schultz, and O. Gutfleisch, J. Alloys Compd. 493, 281 (2010).

${ }^{5}$ F. Buchter Z. Łodziana, A. Remhof, O. Friedrichs, A. Borgschulte, Ph. Mauron, A. Züttel, D. Sheptyakov, G. Barkhordarian, R. Bormann, K. Chlopek, M. Fichtner, M. Sørby, M. Riktor, B. Hauback, and S. Orimo, J. Phys. Chem. C 112, 8042 (2008).

${ }^{6}$ Y. Filinchuk, E. Ronnebro, and D. Chandra, Acta Mater. 57, 732 (2009).

${ }^{7}$ F. Buchter et al., J. Phys. Chem. C 113, 17223 (2009).

${ }^{8}$ Y. Filinchuk, D. Chernyshov, and R. Černý, J. Phys. Chem. C 112, 10579 (2008).

${ }^{9}$ Y.-S. Lee, Y. Kim, Y. W. Cho, D. Shapiro, C. Wolverton, and V. Ozolins, Phys. Rev. B 79, 104107 (2009).

${ }^{10}$ K. Chlopek, C. Frommen, A. Leon, O. Zabara, and M. Fichtner, J. Mater. Chem. 17, 3496 (2007).

${ }^{11}$ P. C. H. Mitchell, S. F. Parker, A. J. Ramirez-Cuesta, and J. Tomkinson, Vibrational Spectroscopy with Neutrons with Applications in Chemistry, Biology, Material Sciences and Catalysis (World Scientific, Singapore, 2005).

${ }^{12}$ S. J. Clark et al., Z. Kristallogr. 220, 567 (2005).

${ }^{13}$ K. Refson, P. R. Tulip, and S. J. Clark, Phys. Rev. B 73, 155114 (2006).

${ }^{14}$ M. H. Lee, Ph.D. thesis, Cambridge University, 1995, [http://boson4.phys.tku.edu.tw/qc/mythesis/index.htm].

${ }^{15}$ J. P. Perdew, K. Burke, and M. Ernzerhof, Phys. Rev. Lett. 77, 3865 (1996).

${ }^{16}$ S. Baroni et al., Rev. Mod. Phys. 73, 515 (2001).
${ }^{17}$ A. J. Ramirez-Cuesta, Comput. Phys. Commun. 157, 226 (2004).

${ }^{18}$ E. H. Majzoub and E. Rönnebro, J. Phys. Chem. C 113, 3352 (2009).

${ }^{19}$ T. J. Frankcombe, J. Phys. Chem. C 114, 9503 (2010).

${ }^{20}$ See supplemental material at [http://link.aps.org/supplemental/ 10.1103/PhysRevB.83.024102] for cif files containing crystallographic information and *.xyz files containing the frequencies and amplitudes of the calculated vibrations. The latter may be visualized using jmol: [http://jmol.sourceforge.net/download/].

${ }^{21}$ N. L. Allan, G. D. Barrera, R. M. Fracchia, M. Y. Lavrentiev, M. B. Taylor, I. T. Todorov, and J. A. Purton, Phys. Rev. B 63, 094203 (2001)

${ }^{22}$ G. D. Barrera, D. Colognesi, P. C. H. Mitchell, and A. J. RamirezCuesta, Chem. Phys. 317, 119 (2005).

${ }^{23} \mathrm{An}$ additional contribution to the entropy may be due to disorder, which is neglected here.

${ }^{24}$ A. Liu, S. Xie, S. Dabiran-Zohoory, and Y. Song, J. Phys. Chem. C 114, 11635 (2010).

${ }^{25}$ C. Rongeat, I. Lindemann, A. Borgschulte, L. Schultz, and O. Gutfleisch, Int. J. Hydr. Energy, doi:10.1016/j.ijhydene.2010. 10.008 .

${ }^{26}$ J. Dawidowski, F. J. Bermejo, and J. R. Granada, Phys. Rev. B 58, 706 (1998).

${ }^{27}$ M. Fichtner, K. Chlopek, M. Longhini, and H. Hagemann, J. Phys. Chem. C 112, 11575 (2008).

${ }^{28}$ J. Tomkinson and T. C. Waddington, J. Chem. Soc. Faraday 72, 528 (1976).

${ }^{29}$ A. I. Kolesnikov, I. Natkaniec, V. E. Antonov, I. T. Belash, V. K. Fedotov, J. Krawczyk, J. Mayer, and E. G. Ponyatovsky, Physica B 174, 257 (1991).

${ }^{30}$ A. I. Kolesnikov, V. E. Antonov, Y. E. Markushkin, I. Natkaniec, and M. K. Sakharov, Phys. Rev. B 76, 064302 (2007).

${ }^{31}$ K. Miwa, M. Aoki, T. Noritake, N. Ohba, Y. Nakamori, S. I. Towata, A. Zuttel, and S. I. Orimo, Phys. Rev. B 74, 155122 (2006). 